\title{
Lumen
}

Selected Proceedings from the Canadian Society for Eighteenth-Century Studies

\section{Piron, ou l'apothéose du poète qui ne fut rien}

\section{Stéphanie Loubère}

Volume 35, 2016

URI : https://id.erudit.org/iderudit/1035917ar

DOI : https://doi.org/10.7202/1035917ar

Aller au sommaire du numéro

Éditeur(s)

Canadian Society for Eighteenth-Century Studies / Société canadienne d'étude du dix-huitième siècle

ISSN

1209-3696 (imprimé)

1927-8284 (numérique)

Découvrir la revue

Citer cet article

Loubère, S. (2016). Piron, ou l'apothéose du poète qui ne fut rien. Lumen, 35,

1-17. https://doi.org/10.7202/1035917ar d'utilisation que vous pouvez consulter en ligne.

https://apropos.erudit.org/fr/usagers/politique-dutilisation/ 


\title{
Piron, ou l'apothéose du poète qui ne fut rien
}

\author{
StÉPhanie Loubère \\ Université Paris-Sorbonne
}

\begin{abstract}
À l'âge classique, la figure du poète se partage volontiers entre deux imaginaires: l'ami des Muses (le poète couronné, volontiers mondain, parfois courtisan) ou le pauvre diable (le poète crotté, le rimailleur indigent tenu à l'écart de la société). Ce partage de l'imaginaire associé au poète s'explique par l'évolution qui atteint de façon plus générale le statut de l'écrivain, évolution qui a été l'objet d'études connues, notamment celle de P. Bénichou et sa mise en évidence du «sacre de l'écrivain », ou celle d'A. Viala qui décrit les conditions qui ont présidé à la «naissance de l'écrivainn" ${ }^{1}$. Le milieu du XVIII ${ }^{\mathrm{e}}$ siècle se présente comme un moment où ces deux modes d'inscription dans les espaces littéraire et social (par inclusion ou exclusion - soit «mandarins » soit «bohèmes » pour reprendre la partition de R. Darnton²) se distinguent avec netteté, mais aussi où leur polarité n'est pas stabilisée. Le poète mondain peut ainsi être célébré comme tel, comme un auteur qui par son art a su plaire et rejoindre les exigences du bon goût, qui sait évoluer dans une société capable d'apprécier ses talents et dont la poésie reflète avec élégance l'esprit et la galanterie des salons qu'il fréquente, mais cela n'empêche pas qu'à l'occasion on dénonce en lui le goût
\end{abstract}

1. Paul Bénichou, Le Sacre de l'écrivain, 1750-1830. Essai sur l'avènement d'un pouvoir spirituel laïque dans la France moderne, Paris, Corti, 1985 et Alain Viala, Naissance de l'écrivain. Sociologie de la littérature à l'âge classique, Paris, Minuit, 1985.

2. Robert Darnton, Bohème littéraire et Révolution: le monde des livres au XVIII siècle, Paris, Gallimard, 1983. 
mauvais du poète poudré, sa poésie soluble dans la pratique courtisane ou réduite à un jeu d'esprit frivole et stérile.

Entre le poète décrié, figure ridiculisée, et le poète célébré, heureusement intégré dans la société, il n'est pas toujours aisé de fixer les limites, comme il n'est pas toujours facile, de notre point de vue rétrospectif, d'apprécier la place occupée par les poètes d'alors dans la société civile et littéraire comme dans l'imaginaire du temps. Je voudrais m'arrêter sur un cas qui est révélateur d'une évolution dans la représentation du poète à ce moment critique. Il s'agit d'Alexis Piron, dont l'œuvre polymorphe nous présente les différentes facettes d'un poète traversé par ces évolutions.

Dans La Métromanie, sa pièce la plus connue, le personnage du poète est au cour de l'intrigue. On a pu voir, dans le personnage central de Damis, la première incarnation dramatique du poète romantique $^{3}$, qui rompait avec la tradition du personnage de poète grotesque (comme on en trouve chez Desmarets, chez Régnier ou Furetière par exemple). Cette lecture me semble devoir être nuancée, car elle escamote en partie les enjeux culturels et sociaux de la mise en fiction du poète. Nous verrons que, chez Piron tout particulièrement, le poète se présente comme une figure en transition, étonnante par sa plasticité, mêlant grotesque et idéal et développant une réflexion militante sur le rôle du poète dans la société contemporaine.

\section{La Métromanie de Piron: figure du poète en transition}

Contemporain de Voltaire, Piron (1689-1773) traverse le siècle en épousant de son œuvre protéiforme ses mutations et soubresauts. Polygraphe curieux, poète et dramaturge avant tout, il est célèbre pour son esprit mordant et sa virtuosité implacable d'auteur d'épigrammes. Il perce en composant des pièces pour le théâtre de la Foire, puis fait jouer quelques pièces à la Comédie-Française, dont La Métromanie (1738), qui connut un beau succès, et que l'on considère généralement comme son chef-d'œuvre, mais aussi des tragédies: Gustave Wasa (1734), Fernand Cortès (1744). Il est reçu à l'Académie française en 1753 mais Louis XV refuse de ratifier son élection, pliant sous la pression de

3. C'est le point de vue formulé par Jacques Truchet dans la notice qu'il consacre à la pièce de Piron dans le tome I du Théâtre du XVIII siècle, Paris, Gallimard, coll. «Pléiade», 1972. 
ses adversaires qui font valoir la nature scandaleuse d'une œuvre de jeunesse peu compatible avec la dignité académique: l'Ode à Priape, qui s'ouvrait sur une énergique invocation au «Foutre des neuf garces du Pinde» et où, congédiant Apollon pour Priape, il redonnait toute sa vigueur à la théorie de l'enthousiasme:

Priape soutiens mon haleine,

Et pour un moment dans ma veine,

Porte le feu de tes couillons ${ }^{4}$.

Cet épisode fâcheux lui inspira l'épitaphe qui fit plus pour son immortalité que ne l'aurait sans doute fait son entrée à l'Académie:

Ci gît Piron qui ne fut rien

Pas même académicien 5 .

La formule mérite d'être commentée, notamment pour ce qu'elle occulte de l'entre-deux dans lequel évolua Piron : il fut poète de métier, capable de vivre de ses succès au théâtre, mais n'était cependant pas tout à fait dégagé du système du mécénat. S'il n’a pas profité de la reconnaissance et des bénéfices que procurait l’Académie française, il n'était par pour autant exclu du circuit officiel, puisqu'il fut membre de l'Académie de Dijon, et eut des protecteurs: le comte de Livry lui fit une pension et mit à sa disposition un appartement dans son château, et il obtint plus tard une pension allouée par Louis XV sur l'intercession de Mme de Pompadour. Poète et penseur de la poésie de son temps, il a mené une réflexion suivie sur le rôle imparti au poète, de façon opiniâtre même si indirecte: il n'a pas écrit d'art poétique ni d'ouvrages théoriques, mais le personnage de l'auteur et du poète est l'objet de scrutations et descriptions multiples dans toute son œuvre. La Métromanie, parce qu'elle cristallise toutes ces réflexions, est particulièrement révélatrice de la vision que Piron avait de son métier de poète et mérite pour cette raison qu'on s'y arrête.

4. Alexis Piron, «Ode à Priape», Euvres complètes illustrées, éd. de P. Dufay, Paris, Guillot, 1928-1931, t. X, p. 123. Nous renverrons à cette édition (dorénavant OC), pour toutes les œuvres hors La Métromanie, pour laquelle nous suivrons l'édition de J. Truchet (dans le Théatre du XVIII siècle, op. cit.), conforme à la version originale de $173^{8}$.

5. «Mon épitaphe, épigramme», OC, t. IX, p. 47. 


\section{Histoire de la pièce : l'affaire Antoinette Malcrais de la Vigne}

La comédie de Piron est ancrée dans l'histoire des mœurs du siècle, puisqu'elle fait suite à ce que l'on a appelé l'«affaire Malcrais de la Vigne», mais elle est aussi tournée vers un idéal qui s'en démarque: Piron y décrit les effets d'une vocation dévorante et postule un idéal de liberté et d'autonomie du poète. À partir de 1729 avaient paru dans le Mercure des poésies signées Antoinette Malcrais de la Vigne, présentée comme une demoiselle bretonne. Des admirateurs écrivirent au journal pour louer la poétesse, Voltaire même se laissa aller à lui écrire une épître où il vante sa «muse divine ${ }^{6} »$. Derrière ce pseudonyme se cachait en réalité Paul Desforges-Maillard qui avait imaginé cette supercherie pour faire remarquer ses vers. La supercherie, une fois révélée, fit beaucoup rire. Piron s'empara de cet événement qui avait remué le cercle des gens de lettres pour écrire sa pièce, égratignant au passage Voltaire et tous ceux qui s'étaient laissé mystifier.

Cette pièce est tout entière organisée autour de la figure du poète - des poètes même comme on le verra, et la poésie y joue un rôle essentiel, puisqu'elle est le principal ressort dramatique et comique de l'intrigue. La scène se passe dans la demeure de M. Francaleu, poète amateur pris sur le tard par la folie des vers et qui publie dans le Mercure sous un nom d'emprunt (Mlle de Mériadec de Kersic). Autour de lui, gravite Damis, autre «métromane», un poète qui cherche à percer et qui est tombé sous le charme de cette Bretonne inconnue dont il lit les vers dans Le Mercure. Damis se trouve à un moment critique de sa carrière: sa première pièce doit être jouée ce jour, et décidera de son sort. Son oncle Baliveau (capitoul, compère de Francaleu) menace en effet de lui couper les vivres et même de le faire enfermer s'il ne renonce pas au métier des vers. Un autre jeune homme, Dorante, est amoureux de Lucile, la fille que Francaleu a tirée du couvent pour la marier. Il cherche à s'en faire aimer en lui faisant lire des vers composés par Damis. Sa situation est également critique: son projet de mariage est menacé par les effets possibles que la révélation de la véritable identité de l'auteur des vers séducteurs ne manquera pas de provoquer, ainsi que par un obstacle plus matériel (son père est en procès avec Francaleu).

6. Cette ode «À Mademoiselle de Malcrais de la Vigne, du Croisic en Bretagne » fut publiée dans le Mercure de France (mai 1732, p. 917-920). 
Cette intrigue entrelacée autour du motif de la folie des vers ne vise pas seulement à provoquer le rire en faisant du poète un ridicule. Le tempo comique irrésistible sait ménager, dans les dialogues, un espace pour la réflexion polémique sur quelques-uns des sujets qui touchent à la pratique de la poésie et à la représentation du poète dans la société d'alors. Sans renoncer à faire la satire des poètes, La Métromanie offre une tribune à la fois plaisante et incisive aux débats poétiques du temps. On y trouve, réduit à une tête d'épingle, comme un précipité des remous de la Querelle des Anciens et des Modernes. Damis, le jeune poète si sûr de sa valeur, entretient ainsi un rapport ambigu à l'idée d'héritage et de filiation: Sapho et Mme Deshoulières sont convoquées pour suggérer la continuité valorisante qui mène à Mlle de Mériadec $^{7}$, mais une autre représentation de la temporalité littéraire, marquée par la rupture, est subrepticement proposée. À Baliveau qui affirme que les anciens, qui «Moissonnaient à leur aise où l'on glane aujourd'hui », ont tout dit, Damis rétorque :

Ils ont dit, il est vrai, presque tout ce qu'on pense.

Leurs écrits sont des vols qu'ils nous ont faits d'avance;

Mais le remède est simple: il faut faire comme eux.

Ils nous ont dérobé; dérobons nos neveux;

Et, tarissant la source où puise un beau délire,

À tous nos successeurs ne laissons rien à dire.

Un démon triomphant m'élève à cet emploi.

Malheur aux écrivains qui viendront après moi! (III, 7, p. 1079)

L'argumentation est originale par sa radicalité, et parce qu'elle contre les Anciens sans leur opposer l'idée de continuum, de temporalité linéaire vers le progrès. La conquête de la parole poétique, décrite comme un rapt fait à la postérité, fait émerger une catégorie esthétique promise à un grand succès vers la fin du siècle, mais dont la valorisation reste encore timide: l'originalité. «C'est que cela jamais n'a rien dit comme un autre» (V, 4, p. 1115) s'exclame Francaleu pour dire toute l'admiration qu'il éprouve pour Damis, et s'il lui faut vanter ses propres vers, il évoque «la muse originale / Qui de ses impromptus tous les mois nous régale» $(\mathrm{V}, 6$, p. 1122). L'exaltation ou la prétention du poète

7. Mondor: «De qui parlez-vous donc, Monsieur?»/Damis: «D’une Sapho / D’un prodige qui doit, aidé de mes lumières, / Effacer, quelque jour, l'illustre Deshoulières » (II, 8, p. 1063). 
peut prêter à rire, mais jamais Piron ne lui ôte tout à fait la part de dignité que lui confère une pratique de son art consciente de ses enjeux et de sa valeur. La Métromanie offre donc un tableau qui, pour être comique, n'en reste pas moins toujours nuancé de la place du poète dans la société de son temps: à les observer de plus près, le portrait qu'il propose du poète, le rôle qu'il lui reconnaît et l'ethos particulier qui émane de sa vision ambivalente peuvent nous en convaincre.

\section{Le portrait du poète: un point de vue panoramique et dramatique}

La Métromanie n'offre pas un portrait univoque du poète: le spectateur se voit régalé d'une série de figures loufoques ou sérieuses, dans un inventaire qui diffracte l'image fixe qu'on attendrait d'après le sous-titre de la pièce (La Métromanie ou le poète). Le "métromane», saisi par la folie des vers, y prend ainsi deux visages: celui du poète amateur (Francaleu) et celui du poète de vocation (Damis), qui prêtent tous deux le flanc à la satire, sans être pourtant réduits à des personnages ridicules. On voit aussi un faux poète traverser la scène (Dorante, lointain précurseur du Christian à qui le Cyrano de Rostand prêtera ses talents), et l'on y convoque la fantomatique présence de la (fausse) poétesse du Mercure (la Sapho de Bretagne, masque de Francaleu). Dans cette galerie de portraits du poète, on retrouve la polarisation évoquée précédemment: le poète y est alternativement célébré comme un ami des Muses et comme un «visionnaire » promis à la misère et méritant d'être enfermé. Surtout, la pièce offre un portrait pluriel, prismatique, étonnamment nuancé grâce aux différents points de vue adoptés. Le premier portrait de poète, celui de Damis, est brossé par son valet dans la scène d'exposition, et propose la vision attendue du poète absorbé par sa manie, marginal extravagant dont le labeur consiste à ne rien faire :

\section{MONDOR}

D'état, il n'en a point, il n'en aura jamais.

C'est un homme isolé qui vit en volontaire;

Qui n'est bourgeois, abbé, robin, ni militaire;

Qui va, vient, veille, sue, et, se tourmentant bien,

Travaille nuit et jour, et ne fait jamais rien.

Au surplus, rassemblant, dans sa seul personne, 
Plusieurs originaux qu'au théâtre on nous donne:

Misanthrope, Étourdi, Complaisant, Glorieux,

Distrait... ce dernier-ci le désigne le mieux.

Tenez, s'il est ici, je gage mes oreilles

Qu'il est dans quelque allée, à bayer aux corneilles,

S'approchant, pas à pas, d'un fossé qui l'attend,

Et qu'il n'apercevra qu'en s'y précipitant.

\section{LISETTE}

Mais... mais je m’oriente au portrait que vous faites.

N'est-ce pas de ces gens que l'on nomme poètes? (I, 1, p. 1018)

Le métromane rejoint ici la troupe des ridicules de comédie, un «volontaire ${ }^{8}$ » accaparé par son art, un «distrait» que son idée fixe rendrait impropre à la vie en société. Mondor rejoint ici l'idée courante qui tourne la vocation poétique en «extravagance» risible: Furetière définit le distrait comme «celui qui n'a point d'attention à ce qu'on lui dit, qui ne répond que longtemps après » et précise que «ces sortes de gens sont poètes, ou extravagants ». La folie et la distraction, attributs communs du poète le prédisposent à porter le masque comique. Un second portrait, lui aussi négatif, est donné plus loin par Baliveau, préoccupé par la situation de son neveu Damis qu'il voudrait voir embrasser un «état» plus lucratif que celui d'amant des Muses. C'est le point de vue du philistin, qui reproduit le topos du poète crotté, véritable rebut de la société:

\section{BALIVEAU}

Il versifiera donc! Le beau genre de vie!

Ne se rendre fameux qu'à force de folie!

Etre, pour ainsi dire, un homme hors des rangs,

Et le jouet titré des petits et des grands!

Examinez les gens du métier qu'il embrasse.

La paresse ou l'orgueil en ont produit la race.

[...]

Damis eût figuré dans un poste honorable;

Mais ce ne sera plus qu’un gueux, qu’un misérable. (V, 4, p. 1118)

8. «Volontaire: [...] se dit aussi d'un opiniâtre, d'un fainéant, qui ne veut rien valoir, qui ne veut que ce qu'il veut. Les pères sont malheureux, qui ont des enfants volontaires et libertins, qui ne veulent pas suivre les desseins qu'ils ont pour leur fortune. » (Dictionnaire universel de Furetière) 
La charge prend une autre tournure et une autre saveur lorsqu'elle est mise dans la bouche de ce même Damis, qui passe momentanément du côté des rieurs, de ceux dont la raison répugne à suivre la folie importune des rimailleurs. Son portrait de Francaleu n'épargne guère celui qui partage pourtant avec lui l'amour des vers:

C'est un fort galant homme, excellent caractère,

Bon ami, bon mari, bon citoyen, bon père;

Mais à l'humanité, si parfait que l'on fût,

Toujours, par quelque faible, on paya le tribut.

Le sien est de vouloir rimer malgré Minerve,

De s'être, en cheveux gris, avisé de sa verve,

Si l'on peut nommer verve une démangeaison

Qui fait honte à la rime, ainsi qu'à la raison.

Et malheureusement, ce qui vicie abonde;

Du torrent de ses vers sans cesse il nous inonde.

Le premier il en raille, et souvent s'avilit;

Grimace! Lauteur perce; il les lit, les relit,

Prétend qu'ils fassent rire, et, pour peu qu'on en rie,

Le poignard sur la gorge, en fait prendre copie,

Rentre en fougue, s'acharne impitoyablement,

Et charmé du flatteur, le paie en l'assommant.

$[\ldots]$

Le voici. Tout le corps me frissonne à l'approche

Du griffonnage affreux qu' il a toujours en poche. (I, 3, p. 1029)

Il ne s'agit pas seulement ici pour Piron de répartir les tares que provoque la manie des vers sur ses deux personnages de poètes, pour distinguer l'extravagant paresseux du grotesque fâcheux. Il suggère également que la métromanie n'est pas réductible à un ridicule, ni le métromane à un type comique. Capable de jugement et de lucidité, Damis rappelle aux spectateurs à quel prix estimer la «verve» et la «fougue» des poètes dignes de ce nom. À côté de ces portraits qui désignent le poète comme objet de risée ou de mépris, se font ainsi entendre d'autres voix, qui lui donnent une épaisseur et une complexité inattendue. Piron donne au poète l'occasion de se défendre: l'autoportrait du poète vient redessiner les contours du personnage auquel il menaçait d'être consigné. Cet autoportrait, pour plus de nuances, est également double. Il y a celui du poète rimailleur, qui ignorant ceux qui le «traitent [...] de poète à la douzaine », affiche sa 
prétention de faire des vers «sans peine » et se réjouit de la notoriété qu'il a tout de même acquise sous son masque:

Mais, sous un autre nom, ma muse, en tapinois

Se fait, dans le Mercure, applaudir tous les mois.

$[\ldots]$

J'y prends le nom d'une Basse-Bretonne.

Sous ce voile étranger, je ris, je plais, j’étonne;

Et le masque femelle, agaçant le lecteur,

De tel qui m’eût raillé fait mon adorateur. (II, 1, p. 1042)

L'autosatisfaction presque enfantine de Francaleu se double d'une capacité à porter un regard satirique sur le monde des lettres, et cette lucidité le fait échapper à la caractérisation unidimensionnelle d'un type de comédie. Plus intéressant encore est l'autoportrait du poète inspiré par Damis, qui oblige également le spectateur à réajuster sa perception du personnage. Le poète décrit ainsi son «baptême » poétique, qui lui vaut le surnom de M. de l'Empyrée:

On tenait table encore; on se serre pour nous.

La joie, en circulant, me gagne ainsi qu'eux tous;

Je le sens, j'entre en verve, et le feu prend aux poudres.

Il part de moi des traits, des éclairs et des foudres;

J'ai le vol si rapide et si prodigieux

Qu'à me suivre on se perd, après moi, dans les cieux;

Et c'est là qu'à grands cris je reçois des convives

Ce nom qui va du Pinde enrichir les archives. (I, 6, p. 1034)

Ce récit autorise bien sûr une lecture satirique (qui nous dit que la fougue de Damis n'est pas à l'image de celle de Francaleu?), mais le reste de la pièce invite à envisager la réalité des talents de Damis, dont on sait par exemple que les vers ont su charmer la belle Lucile, et dont la pièce ne tombe, à la fin de l'intrigue, que sous la pression d'une cabale. On comprend que le texte ait conduit à voir en Damis le précurseur des poètes maudits du siècle suivant, le cœur pur que rien ne détourne de son idéal, et qui porte haut l'ambition de son génie:

Le nourrisson du Pinde, ainsi que le guerrier,

À tout l'or du Pérou préfère un beau laurier.

$[\ldots]$

Qu'on me laisse, à mon gré, n’aspirant qu'à la gloire,

Des titres du Parnasse anoblir ma mémoire, 
$[\ldots]$

Ma vertu donc se borne au mépris des richesses,

À chanter des héros de toutes les espèces,

À sauver, s'il se peut, par mes travaux constants,

Et leurs noms et le mien des injures du temps. (III, 7, p. 1077-1078)

Ce n'est pas la seule lecture possible, mais elle vient donner un relief nouveau à la figure du poète, qui déborde de la simple dimension comique. Ce paradoxe soigneusement cultivé qui permet de marier le grotesque et le génie pour faire le portrait du poète se retrouve dans la dernière forme d'autoportrait que constitue l'écriture autobiographique, pratiquée ici ou là par Piron, notamment dans la préface de sa pièce, mais aussi en d'autres lieux de son œuvre - notamment L'Apothéose de Binbin, texte sur lequel nous reviendrons. Dans la préface qui accompagne la Métromanie dans certaines éditions à partir de $175^{9}$, il se présente comme un double possible de Damis, dont les talents, même véritables, peuvent prêter à sourire :

Je ne me donne que pour ce que je suis, que pour un de ces esprits trop ordinaires qui reçoivent le jour, non sous l'astre bénin dont l'influence est si rare, mais sous cet astre pestilentiel et non moins dominant qui fait qu'on a la fureur d'être poète, et souvent, qui pis est, celle encore de se le croire.

Je cédai donc à la force majeure : ainsi peut bien s'appeler cette manie qui fait ici tout à la fois l'excuse bonne ou mauvaise de l'auteur, et le titre de la pièce, et je lui cédai d'autant plus naturellement, qu'après tout le bien et le mal qu'elle m’a causé, je ne pouvais manquer d'avoir une vive démangeaison d'en dire tout le mal et le bien que j'en pense ${ }^{10}$.

L'œuvre de Piron donne ainsi une idée nuancée de la façon dont les poètes étaient perçus par la société et dont ils se voyaient euxmêmes, avec lucidité, autodérision, mais aussi beaucoup de fierté et de grandes aspirations.

Cela se vérifie lorsque l'on considère un autre point débattu de façon insistante dans la trame de la fiction dramatique : l'utilité de la poésie. Si, comme le relève justement I. Martin, «dans toute l'œuvre

9. Cette longue préface dans laquelle Piron confie l'histoire de sa propre vocation poétique est absente de la plupart des éditions, et notamment de l'édition originale. Elle est présente dans les Euvres publiées en 1758, 1764 et 1766, et reproduite par Dufay dans les OC.

10. OC, t. III, p. 11. 
de Piron le thème de l'impossibilité d'être à la fois un poète passionné et riche revient de manière obsessionnelle ${ }^{11}$ ", cela ne l'empêche pas de défendre dans plusieurs pièces l'utilité (morale mais aussi sociale) de l'auteur de théâtre et, plus largement, du poète.

Lutilité morale de la poésie est fièrement défendue par Damis dans la pièce, notamment lorsqu'il prétend «instruire tout Paris» de sa vertu:

Comment? Par mes écrits.

Je veux que la vertu plus que l'esprit y brille.

La mère en prescrira la lecture à sa fille. (III, 7, p. 1080)

La formule aura un succès qui nous renseigne sur la fortune de la pièce: Sylvain Maréchal en orne, sous une forme détournée ( «Lamant en prescrira la lecture à sa mie ») la page de titre de sa Bibliothèque des amants $\left(1777^{12}\right.$ ), un libelle anonyme (Les Fureurs utérines, $1791^{13}$ ) en inverse la formule ( «La mère en proscrira la lecture à sa fille ») et Sade en fait malicieusement l'épigraphe de sa Philosophie dans le boudoir (1795). La prétention de Damis n'est donc pas vaine: toutes ces refontes saluent, à leur façon parfois irrévérencieuse, l'efficacité de la parole poétique (celle de Piron en l'occurrence) pour fixer durablement idées et principes. Mais nous intéresse ici tout spécialement, l'idée de l'utilité sociale, qui revient comme un leitmotiv dans toute l'œuvre de Piron. Il donne régulièrement la parole aux détracteurs du métier poétique, bourgeois et pères inquiets pour leur progéniture qui demandent à quoi servent les poètes, à l'exemple de Baliveau:

Que font-ils pour l'état? Pour les leurs? Pour eux-mêmes?

De la société véritables frelons,

Chacun les y méprise ou craint leurs aiguillons. (V, 4, p. 1118)

ou du père du poète dans Le Caprice, un opéra comique composé par Piron en 1724:

11. Isabelle Martin, «Enquête du personnage de l'auteur chez Alexis Piron », La Commedia dell'arte, le théâtre forain et les spectacles de plein air en Europe, $X V I^{e}$ XVIII siècles, Paris, Klincksieck, 1998, p. 131.

12. Sylvain Maréchal, Bibliothèque des amants, Odes érotiques, Paris, Duchêne, s.d. [1777].

13. Les Fureurs utérines de Marie-Antoinette, femme de Louis XVI, Au Manège et dans tous les Bordels de Paris, s.n., 1791. 
LE POÈTE

Air: Il faut que je file file

Est-il rien de plus utile

Qu'un bon rimeur, à l'état

Il répand sa docte bile,

Sur le vice avec éclat;

Et pour les mours d'une ville

Sert plus qu'un bon magistrat.

Est-il rien de plus utile

Qu'un bon rimeur à l'état?

LE PÈRE

Tu ne t'ôteras pas ces maudites visions-là de la tête! Regarde, malheureux, regarde ton frère le médecin et ton cousin l'avocat: voilà des gens utiles à la République, cela ${ }^{14}$ !

Dans la préface de La Métromanie, Piron revient sur la question avec une modestie calculée, que le succès de sa pièce permet d'inverser en apologie subreptice. Dans le sillage des maximes désabusées d'un Malherbe défendant malgré tout la profession poétique en convoquant l'image du joueur de quille, il déclare :

Je n'entretiens mon lecteur de si petites choses, et n'ose parler de moi si longtemps contre la loi du sage, qu'en vue de me justifier humblement devant la société dont bientôt je me sépare dans un âge avancé, sans avoir eu le bonheur de lui pouvoir être utile et nécessaire, n’ayant labouré, bâti, calculé, médicamenté, plaidé, jugé, prêché, ni combattu; n'ayant fait pour elle en un mot que des vers; et quels vers encore? Des vers comme on vient de le voir, moins inspirés par Minerve que par la nécessité15.

Il développe la même idée dans la version longue de son épitaphe, en avançant l'idée paradoxale d'une inutilité mémorable:

MON ÉPITAPHE, ÉPIGRAMME.

Ci gît... Qui? Quoi? Ma foi, personne, rien.

Un qui vivant, ne fut valet ni maître,

Juge, artisan, marchand, praticien,

Homme des champs, soldat, robin ni prêtre,

Marguillier, même académicien,

Ni frimaçon. Il ne voulut rien être,

14. OC, t. IV, scène 11, p. 269-270.

15. Ibid., t. III, p. 21. 
Et vécut nul: en quoi certes il fit bien;

Car après tout, bien fou qui se propose,

Venu de rien, et revenant à rien,

D'être en passant ici-bas quelque chose!

Pour le soulagement des mémoires, et pour le mieux,

J'ai cru devoir réduire cette épitaphe à deux vers.

Ci gît Piron qui ne fut rien

Pas même académicien ${ }^{16}$.

Ce paradoxe nous conduit à considérer pour finir la très singulière ambivalence du point de vue de Piron sur son ethos de poète. Le désir de justifier son état de poète est permanent dans son œuvre, et oblige son lecteur à partager son point de vue fluctuant. Il y a de l'arrogance dans l'humilité qu'il affiche, et un peu de dérision dans son orgueil de poète. Le sentiment profond de sa vocation poétique n’exclut pas le regard extérieur, volontiers critique, de la société à laquelle il appartient. La satire et la profession de foi poétique ont chez lui des accointances étonnantes. Se côtoient ainsi chez lui l'effacement et l'amour-propre: d'un côté il est celui «qui ne fut rien », ou «Binbin » (diminutif de Bénigne, son nom Bourguignon qu'il rappelle volontiers dans ses lettres et diverses œuvres où il se met en scène); d'un autre, il revendique la noblesse du métier de poète. Rigoley de Juvigny, son biographe et éditeur testamentaire, rapporte dans la Vie de Piron qui accompagne l'édition de ses Euvres, plusieurs anecdotes où Piron affiche sa fierté de poète. C'est ainsi qu'après avoir passé une joyeuse soirée avec ses amis les poètes Gallet et Collé, Piron aurait été arrêté par le guet, et interrogé par un commissaire:

«Qui êtes-vous? votre nom?»- «Piron»- «Quel est votre état?» «Poète. »- «Poète?»- «Oui, monsieur, poète. Eh! où donc vivez-vous pour ne pas connaître le poète Piron? [...] Oui, monsieur, mon état est d'être poète, état le plus grand, le plus noble, le plus sublime que les hommes puissent embrasser, quand c'est du génie qu'ils le tiennent ${ }^{17}$ ! »

Si le regard que porte Piron sur les poètes peut être un brin cynique, jamais il ne rabat la «noblesse » irréductible de leur état. Nul n’échappe

16. Ibid., t. IX, p. 47.

17. «Vie d'Alexis Piron», CEuvres complètes d'Alexis Piron, publiées par M. Rigoley de Juvigny, Paris, Lambert, 1776, t. I, p. 74-75. 
à cette admiration mêlée de sarcasme - pas même lui, ainsi que nous le révèle une lettre écrite à un ami :

J'ai, par dessus vous, trois grands avantages de nature: folie, gueuserie et vanité; trois bases du noble métier des vers, sans lesquelles on passe tout doucement son chemin comme d'honnêtes gens, sans rats, sans faute et sans poux; et je ne donnerais pas un fétu de qui s'embarque sur la mer d'Hippocrène, sans cette cargaison ${ }^{18}$.

Gueuserie et noblesse sont les attributs contrastés du poète selon Piron, à qui le mouvement de fierté autocritique permet de trouver sa place à la fois dans l'espace imaginaire du Parnasse et dans une société dont il accepte lui aussi et malgré tout les règles en «honnête homme ». On ne s'étonne pas alors que dans son œuvre se juxtaposent également la raillerie et l'éloge, jusqu'à parfois se confondre. Dans La Métromanie, Damis a pour surnom M. de l'Empyrée, et l'onomastique ici concentre toute l'ambiguiité du portrait, puisqu'une double interprétation de ce nom est possible : il peut renvoyer à la prétention déplacée du mauvais poète, à un snobisme ridicule comparable à celui du M. de la Souche de Molière, mais il permet pourtant aussi d'esquisser le portrait idéalisé du vrai poète, avec l'anoblissement symbolique que lui confère son génie. On peut suivre dans la pièce toutes les variations sur le thème de la «noblesse » du poète, qu'elle soit vantée chez Damis par Francaleu:

Ce jeune homme n'est pas du commun des humains.

Les grands seigneurs déjà se l'arrachent des mains.

$[\ldots]$

Mais apprenez de moi qu'un ouvrage d'éclat

Anoblit bien autant que le capitoulat. (V, 4, p. 1116 et 1119. Nous soulignons)

ou par Damis, lorsqu'il s'imagine pouvoir prétendre à l'immortalité due aux grands hommes:

Le nourrisson du Pinde, ainsi que le guerrier,

À tout l'or du Pérou préfère un beau laurier.

L'avocat se peut-il égaler au poète?

De ce dernier la gloire est durable et complète.

Il vit longtemps après que l'autre a disparu.

Scarron même l'emporte aujourd'hui sur Patru.

18. Lettre à l'abbé Legendre, 7 novembre 1728, dans Alexis Piron épistolier, éd. de G. von Proschwitz, Göteborg, Acta Universitatis Gothoburgensis, 1982, p. 68. 
[...]

Qu'on me laisse à mon gré, n’aspirant qu'à la gloire,

Des titres du Parnasse anoblir ma mémoire,

Et primer dans un art plus au-dessus du droit,

Plus grave, plus sensé, plus noble qu'on ne croit. (III, 7, p. 1077. Nous soulignons)

La préface que Piron adjoint à l'édition de sa pièce est bien dans cet «entre-deux» où le poète est à la fois objet de louange et de sarcasme. Il y décrit en «bien » et en «mal » la vocation du poète, que ce soit la sienne ou celle de son personnage:

En conservant à mon poète quelques petits ridicules essentiels à la profession, je ne l'en ai pas moins fait bon, franc, généreux, brave et désintéressé. [...] M. de l'Empyrée honnêtement fourni des ridicules de son état, ne laisse pas d'être leste, gai, doux, sociable et galant ${ }^{19}$.

Une troisième forme d'ambivalence concernant l'éthos poétique se fait jour: Piron veut redresser l'image du poète dans la société, et se livre à la défense réaliste d'une profession idéaliste (ou, pour être plus précis, en voie d'idéalisation):

Véritablement voyant avec chagrin que dans tous les temps, et chez toutes les nations, les poètes en général étaient livrés à la risée du public par les poètes mêmes, et de plus les voyant taxés par ce public de bien des vices [...], j’avais pris à tâche de présenter sur la scène un poète qui, sans sortir de son caractère singulier, fût une fois fait de façon à nous relever d'un préjugé si peu favorable ${ }^{20}$.

Ailleurs, il ouvre une alternative pour décrire la vocation des poètes:

Sérieusement et par état occupés par le solide, ils n’ont garde de concevoir ni de soupçonner l'héroïsme ou la folie du vrai poète, qui, vis à vis de la misère, pense en parlant de sa muse, comme vis à vis d'un avenir menaçant, en parlant de son fils, pensait Agrippine: Moriar, modo regnet ${ }^{21}$.

L'hésitation entre héroïsme et folie pour qualifier le «vrai» poète ainsi que la comparaison ambiguë avec la mère de Néron nous disent quelle idée intriquée et contrastée Piron se faisait de sa profession,

19. OC, t. III, p. 36-38

20. Ibid., p. $37-38$.

21. Ibid., p. 40. 
donnant de la poésie une idée à la fois sublime et dérisoire, aussi digne de dévotion que d'indifférence. La conscience d'une vocation, d'un idéal, est assourdie par sa mise à distance volontaire, par l'ironie et l'autodérision. Lun des derniers textes de Piron, une pièce en vers intitulée Apothéose de Binbin ou Voyage à l'Empyrée, témoigne de ce paradoxe véritablement constitutif de son identité poétique. À la faveur «d'un vin fumeux et traître» son âme quitte son corps et se trouve changée en «étoile à beau rayon doré22 ». L'autoportrait qui se déploie dans ces vers est continuellement modulé selon ces deux tentations: prendre au sérieux la vocation poétique ou la tourner en dérision. Binbin prend de l'altitude pour observer la terre, rit de la voir si minuscule et sonde le néant dont il a été tiré avant d'être propulsé vers le firmament:

Le voilà donc ce globe terrien,

Dis-je en riant, qu'on s'imagine immense!

Où du néant sorti pour être rien,

Né d'une femme et d'un homme de bien,

Faute de nom, de titres, de chevance,

Et d'y pourvoir n'ayant aucun moyen,

J'arrivai nu et naquis sans naissance ${ }^{23}$.

Le rire de n'avoir été rien laisse place cependant à la fierté de n'être pas l'une des «fausse $[s]$ comète $[s]^{24} »$ qui traversent le ciel. Apercevant Pégase au loin, il rit également de ses ambitions littéraires:

Dans un beau songe, insensé métromane,

Sur ce cheval je me suis cru souvent:

Fier et gaillard, j’allais le nez au vent,

Et je faisais claquer mon fouet profane.

N'étais-je pas (mon siècle en fut témoin)

Un joli Plaute, un bel Aristophane!

Un si doux rêve aussi n'allait pas loin.

Vous voilà donc, noble sang de Méduse,

Dis-je au coursier de l'immortalité !

Sur vous cent fois je me suis cru monté,

Quand le jouet d'une folâtre muse,

22. Apothéose de Binbin ou Voyage de l'Empyrée. À Monsieur le comte de saint Florentin, OC, t. VIII, p. 321 et 322. Piron aurait écrit ces vers à l'âge de 81 ans.

23. Ibid., p. 324.

24. Ibid., p. 327. 
Comme un nigaud qu'aisément on abuse,

Sur chevillard je n'étais que planté25.

Mais c'est ce même Pégase qui l'engage à moins de modestie et à reconnaître les hommages que lui a rendus son public: cette «apothéose de Binbin » n'est pas une parodie et la conscience de sa valeur ne s'y dissout pas, comme on aurait pu s'y attendre, dans le burlesque:

Il dit: mon âme, à ces mots rassurée

Sur quelque bruit que je laisse après moi,

Mon adieu fait, je gagnai l'empyrée,

Où me voici brillant, tranquille et $\operatorname{coi}^{26}$.

La tranquillité de Piron s'affirme comme un état d'équilibre entre affirmation et effacement de soi, le «brillant» et le «coi», qui lui permet de prétendre à l'apothéose tout en savourant son anéantissement. On observe chez lui une ambivalence féconde qui ne conduit pas à un déchirement (la délectation morose du poète maudit), mais à une conscience exigeante, sans illusion, et pourtant résolument souriante des droits et devoirs du poète dans une société prête à l'encenser ou à le dénigrer. Piron ancre son portrait du poète dans la réalité, une réalité moderne, instable, qu'il observe avec un souci presque anthropologique. Il nous offre en fin de compte, dans La Métromanie et dans une bonne partie de son œuvre, un instantané dramatique (à tous les sens du terme) de la place qu'a pu occuper le poète dans une société qui nourrissait à son égard à la fois une terrible méfiance et de grandes ambitions. 